\title{
Multiple Cartilaginous Exostoses Causing Severe Cervical Myelopathy in a Puppy Dog
}

\author{
Mareliza Possa de Menezes ${ }^{1}$ Andréia Coutinho Facin ${ }^{1}$ Larissa Godoi Máximo ${ }^{1}$ Mayara de Cássia Luzzi ${ }^{2}$ \\ Luis Gustavo Gosuen Gonçalves Dias ${ }^{1}$ Bruno Watanabe Minto ${ }^{1}$ \\ ${ }^{1}$ Department of Veterinary Clinic and Surgery, School of Agricultural \\ and Veterinary Sciences, São Paulo State University, Jaboticabal, São \\ Paulo, Brazil \\ 2 Department of Veterinary Pathology, School of Agricultural and \\ Veterinary Sciences, São Paulo State University, Jaboticabal, São \\ Address for correspondence Luis Gustavo Gosuen Gonçalves Dias, \\ PhD, Department of Veterinary Clinic and Surgery, School of \\ Agricultural and Veterinary Sciences, São Paulo State University, \\ Jaboticabal, Via de Acesso Prof. Paulo Donato Castellane s/n, CEP \\ 14884-900, São Paulo, Brazil (e-mail: gustavogosuen@gmail.com).
} Paulo, Brazil

VCOT Open 2021;4:e1-e4.

\author{
Abstract \\ Keywords \\ - multiple cartilaginous \\ exostosis \\ - cervical spinal cord \\ compression \\ - hereditary \\ neurological \\ disorders \\ - osteochondromatosis \\ - atlantoaxial joint \\ stabilization
}

This study aimed to report a case of multiple cartilaginous exostoses (MCE) causing severe cervical myelopathy in a puppy dog. An 80-day-old Dachshund cross weighing $2.2 \mathrm{~kg}$ presented with progressive development of non-ambulatory tetraparesis, proprioceptive ataxia in all four limbs, and cervical hyperesthesia. Multiple calcified masses in the cervical spine at the levels of $\mathrm{C1}-\mathrm{C2}$ and $\mathrm{C4}-\mathrm{C} 7$, with several points of spinal cord compression, were identified. Ventral atlantoaxial fusion was performed. Four days later, four masses were surgically removed by dorsal laminectomy of $\mathrm{C} 1-\mathrm{C} 2$ and $\mathrm{C} 4-\mathrm{C} 7$, and a right facetectomy between $\mathrm{C} 4$ and $\mathrm{C} 5$ was performed. Multiple cartilaginous exostoses were confirmed by histopathological examination. Thirty-six months after surgery, the patient showed no recurrence of neurological signs. Ventral atlantoaxial fusion, and surgical removal of MCE masses followed by decompression was effective on alleviating clinical sings in the short and medium term.

\section{Introduction}

Multiple cartilaginous exostoses (MCE), also known as osteochondromatosis, is a rare hereditary disorder of skeletal development associated with mutation of the genes exostosin 1 or 2 , described in humans, horses, dogs and cats. ${ }^{1-5}$ The excessive cartilaginous proliferation can occur in the metaphysis of long bones causing irregular bone formation on bone surfaces. ${ }^{3,4}$ When MCE masses affect the vertebrae, spinal cord compression and gait disorders may occur. ${ }^{1,4,6}$ The treatment consists of surgical removal of the masses in cases of mechanical impairment. ${ }^{1,4-6}$ This is the first case report of MCE affecting an actively growing puppy dog treated surgically with favourable outcome with two stages surgical procedures.

received

September 22, 2020

accepted after revision

November 15, 2020
DOI https://doi.org/

10.1055/s-0040-1722202.

ISSN 2625-2325.

\section{Materials and Methods}

\section{Case Description}

An 80-day-old male Dachshund cross weighing $2.2 \mathrm{~kg}$ presented to our Veterinary Teaching Hospital with a 30-day history of motor incoordination. Neurological examination revealed an ambulatory tetraparesis with proprioceptive deficit worst in the right pelvic limb, intact flexor withdrawal reflexes, increased patellar tendon reflexes bilaterally and hyperesthesia of the cervical area. All cranial nerve reflexes were intact. Orthopaedic examination findings included pain during hip and shoulder hyperextension bilaterally, and crepitus on palpation of the right elbow and right carpus.

Complete blood count, serum biochemical profile and urinalysis results were within normal limits. The patient

\section{(c) 2021. The Author(s).}

This is an open access article published by Thieme under the terms of the Creative Commons Attribution License, permitting unrestricted use, distribution, and reproduction so long as the original work is properly cited. (https://creativecommons.org/licenses/by/4.0/)

Georg Thieme Verlag KG, Rüdigerstraße 14, 70469 Stuttgart, Germany 
was sedated and radiographic images of the cervical spine were obtained. Radiopaque material with a smooth margin was seen in the intervertebral spaces between the dorsal aspect of $\mathrm{C} 1-\mathrm{C} 2$ and the articular facets of $\mathrm{C} 3-\mathrm{C} 4, \mathrm{C} 4-\mathrm{C} 5, \mathrm{C} 5-$ C6 and C6-C7. There was also a decrease in the width of the intervertebral disc space with a loss of visualization of the articular processes, which was most pronounced in the segments C1-T3 (- Fig. 1A), T6-T10 and L2-S1. Additionally, articular incongruity with sclerosis and irregular margins of the articular surface was seen bilaterally in the shoulder and hip joints, with flattening of the femoral and humeral heads. Enlargement of the distal third of costal arches and costochondral junctions was also observed.

Two weeks later, the dog showed progressive worsening of neurological signs, presenting with non-ambulatory tetraparesis, diminished proprioceptive reactions (particularly on the right thoracic limb) and a diminished flexor withdrawal reflex in the right thoracic limb.

Cervical myelography (iohexol [90 mg/kg / $40.82 \mathrm{mg} / \mathrm{lb}]$ ) revealed dorsal compression of the spinal cord at the atlan-

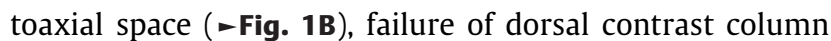
filling between C4 and C7 (- Fig. 1B) and interruption of contrast on the right side of $\mathrm{C} 1-\mathrm{C6}$ (-Fig. 1C).

A staged atlantoaxial ventral fusion ${ }^{7}(2.0 \mathrm{~mm}$ cortical bone screws and polymethylmethacrylate) and 4 days later C1-C2 and C4-C7 dorsal laminectomy ${ }^{8}$ was performed. Autologous cancellous bone graft taken from greater tubercle of the right humerus was applied to enhance atlantoaxial fusion.

At the dorsal aspect of the C1-C2 intervertebral space, a white circular fibroelastic mass of $\sim 2.5 \mathrm{~cm}$ diameter was visualized. Other similar but smaller structures were found in the intervertebral spaces and between the articular processes of C4-C7. After inspection, $90 \%$ of the dorsal arch of $\mathrm{C} 1$ was removed in a caudocranial direction; $75 \%$ of the $\mathrm{C} 2$ dorsal lamina was also removed. The insertion of the nuchal ligament was left intact. The dorsal laminae of C4-C7 and the right articular processes of $\mathrm{C} 4-\mathrm{C} 5$ were also removed.

The spinal cord was then exposed and evaluated. All spinal cord segments were compressed by masses. At C1-C2 and

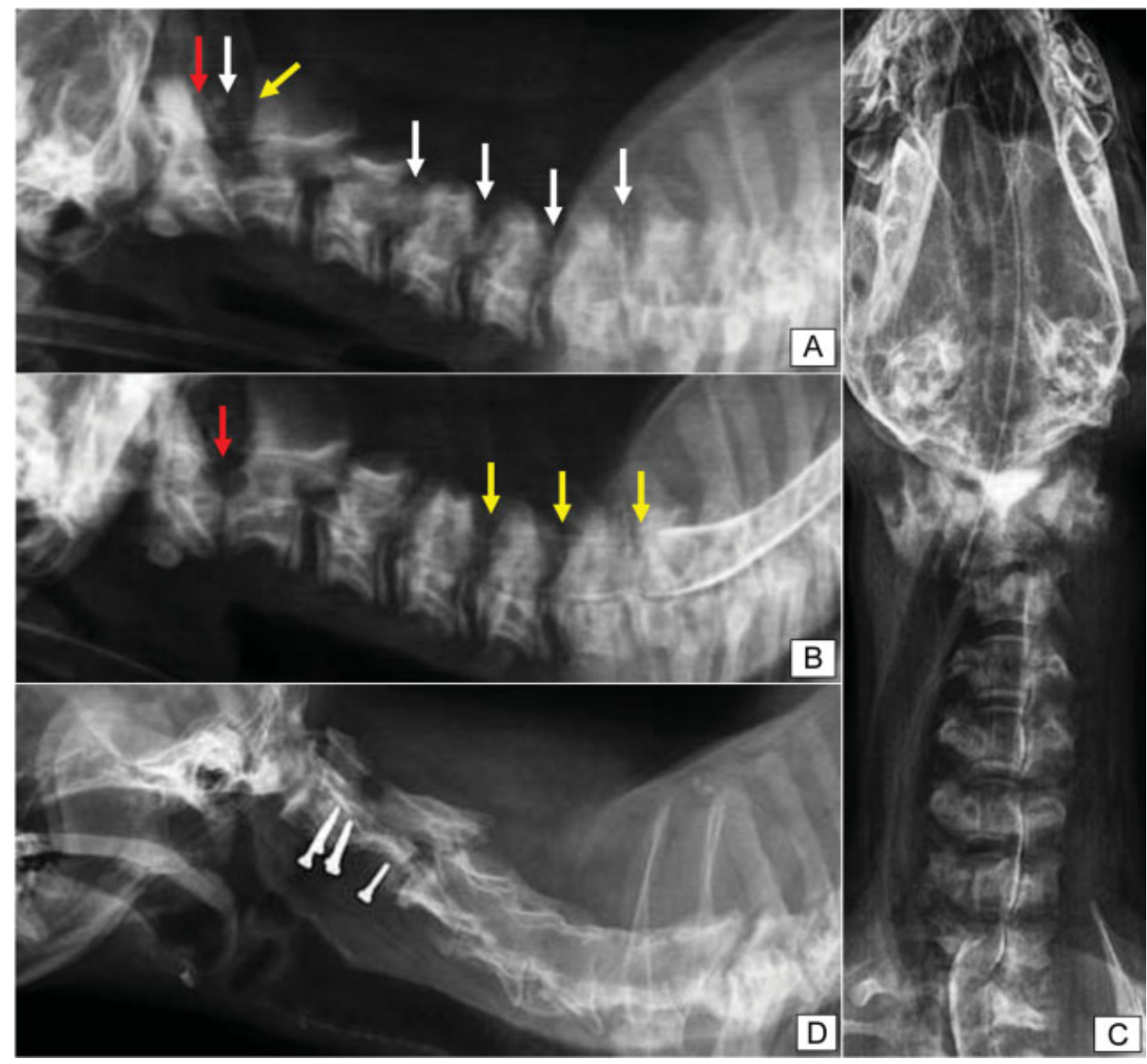

Fig. 1 Lateral and ventrodorsal radiographic views of the cervical spine of an 80-day-old $\operatorname{dog}(A-C)$ and 36 months after surgery (D). (A) A plain radiographic image showing opaque material with a smooth margin on the dorsal aspect of $C 1-C 2$ and the articular facets of $C 3-C 4, C 4-C 5, C 5-$ C6 and C6-C7 (white arrows). Partial osteolysis is visible on the dorsal arch of C1 (red arrow) and the spinous process of C2 (yellow arrow). (B) A myelography image showing disruption of the contrast column due to dorsal spinal cord compression by a proliferative growth at the level of $\mathrm{C} 1$ C2 (red arrow), and a lack of the dorsal contrast column between C4-C7 (yellow arrows) due to further compression points. (C): Ventrodorsal radiographic view (myelography image) showing interruption of contrast on the right side of C1-C6. (D) Radiographic image at 36 months postoperatively showing arthrodesis between $\mathrm{C} 1$ and $\mathrm{C} 2$, absence of listhesis between the cervical vertebrae and fusion of the remaining joint facets as well as the vertebral bodies. 
C5-C6, the spinal cord was purplish and adhered to the mass; this appearance improved immediately after decompression.

\section{Results}

Macroscopically, the exostotic formations were characterized as fibrocartilaginous, irregular, homogeneous tissue with a whitish cut surface. On microscopic examination, the samples were composed of cartilaginous tissue associated with significant fibrosis, foci of necrosis and calcification and areas showing deposition of material with a myxoid aspect. Additionally, a slight increase in density of lymphocytes and plasmocytes was observed. No evidence of malignancy was seen in the samples (-Fig. 2). These findings are supportive of a diagnosis of MCE.

Over the following 3 days after the first procedure, the patient showed minor improvement of neurological signs and, although non-ambulatory tetraparesis was still present, the dog could stand when placed in a quadrupedal position.

The patient was able to stay standing by yourself the day after the second procedure and started to walk on the second postoperative day and improved progressively from this point onwards. Remission of neurological signs was observed progressively; on the 25th day after the last surgical intervention, examination revealed normal proprioceptive reactions in all four limbs, a mild plantigrade and palmigrade position in the limbs (worse on the left side), contracture of the cervical musculature and progressive spondylodesis.

Thirty-six months after surgery, the patient showed no proprioceptive deficits, a mild plantigrade and palmigrade position in the left limbs. Intermittent mild lameness of the left thoracic limb and the pelvic limbs was still present. Total limitation in lateral neck movement, pain on hip and shoulder hyperextension and pain on dorsal extension of the tail were also noted.

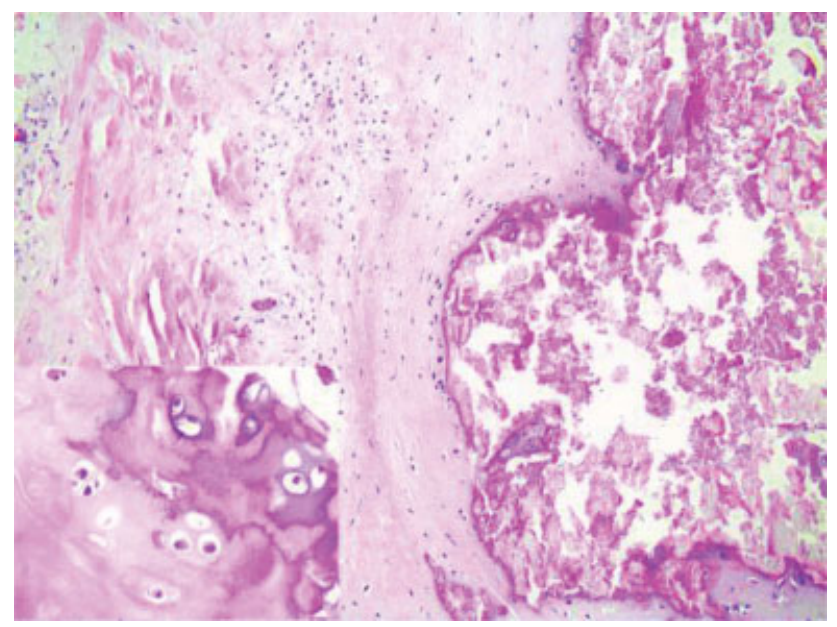

Fig. 2 Photomicrograph of the excised tissue. Histopathology revealed a cartilaginous tissue associated with significant fibrosis covering the bone. Islands of collagen and irregular chondrocytes in cartilage matrix were seen (detail). No evidence of neoplasia was found. 20x obj. lens and detail 40x obj. lens, haematoxylin-eosin.

\section{Discussion}

The condition of MCE has rarely been reported in veterinary patients and it is usually observed in endochondral ossification sites. ${ }^{4,6,9}$ The large mass near the atlantoaxial joint and C4-C7 segment was responsible for the most severe neurological signs in our case, although smaller proliferative cartilaginous lesions were also observed in other regions of the spine (C4-T1; L2-L3; L7-S1), and abnormalities were identified in the hip and shoulder. Similar signs have also been reported in humans, dogs and cats. ${ }^{2,5,9}$

The clinical manifestations of MCE are variable. Most patients are asymptomatic or present with only a few incidental signs. However, despite being considered a benign disease, severe musculoskeletal and neurological dysfunction can result from the compression of vessels and central neurological tissue in MCE. ${ }^{1,3,4,6}$ This was observed in our patient in the form of a dramatic, rapid and severe cervical myelopathy, despite of previous cases reports described MCE as progressive and slow with onset of clinical signs to be after 3 months of age. $3,4,6$

The clinical signs of ambulatory tetraparesis, intact flexor withdrawal reflexes and increased patellar tendon reflexes observed at our patient's first visit are clearly compatible with spinal cord compression between $\mathrm{C} 1$ and $\mathrm{C} 2$. $^{1,6}$ However, this was subsequently followed by the development of lower motor neuron signs, differently from the initially observed upper motor neuron signs. We hypothesized that this was due to rapid growth of the masses affecting the C6T1 segments and therefore impingement of cervical neural foramina.

Histopathological findings in this study included a hyaline cartilage capsule involving the lamellar bone and bone marrow, collagen and cartilage matrix in the tissue and associated areas of calcification; these findings are supportive of a diagnosis of MCE. ${ }^{2-4}$

Following previous recommendations, ${ }^{5-7}$ an extensive and early dorsal laminectomy was successfully performed, including $\mathrm{C} 1-\mathrm{C} 2$ and $\mathrm{C} 4-\mathrm{C} 7$ segments. To our knowledge, this is the largest one-stage cervical laminectomy reported in a dog. This aggressive approach was taken to provide enough decompression at multiple sites and to allow rapid and longterm remission of clinical signs. On the other hand, atlantoaxial joint stabilization was necessary to prevent higher levels of cervical instability. ${ }^{10}$ Despite the full-thickness removal of the dorsal laminae between $\mathrm{C} 4$ and $\mathrm{C} 7$, only right unilateral facetectomy was performed between $\mathrm{C} 4$ and $\mathrm{C} 5$, where a significant macroscopic dorsolateral compression was observed.

Thirty-six months after the surgeries, the patient presented with some mild neurological signs and lameness. We believe that hip and shoulder alterations and nerve root compression can explain those findings. A palmigrade position was not observed in the right thoracic limb probably because the facetectomy was performed only on the right aspect of $\mathrm{C} 4-\mathrm{C} 5$.

Early diagnosis and prompt surgical planning were crucial for the success of treatment in our patient. Myelography 
proved to be a simple, low-cost and useful technique to determine the points of spinal cord compression and plan the surgical procedures. The ventral stabilization of $\mathrm{C} 1-\mathrm{C} 2$, alongside surgical decompression by multiple laminectomies of the C1-C2 and C4-C7 segments and facetectomy between $\mathrm{C} 4$ and $\mathrm{C} 5$, was effective and supported a considerable remission of the neurological signs.

\section{Conclusion}

This is the first case report of an 80-day-old dog with severe clinical signs of MCE and a longer postoperative follow-up period. Despite the aggressive surgical approach, no complications were observed; the patient had a good outcome postsurgery and a high quality of life throughout the 36 months of follow-up.

\section{Authors' Contributions}

M.P.M. and L.G.G.G.D. contributed to the conception of study, study design, acquisition of data, data analysis and interpretation. A.C.F., L.G.M., B.W.M. contributed to the data analysis and interpretation of surgical management and image diagnosis. M.C.L. contributed to the acquisition of data, data analysis and interpretation of histopathological findings. All authors drafted, revised and approved the submitted manuscript.

\section{Conflict of Interest}

None declared.

\section{Acknowledgments}

The authors would like to thank Rosemary Oliveira Vasconcelos DVM, MS, PhD, Department of Veterinary Pathology,
UNESP-FCAV, for essential assistance with histopathological analysis, and Veterinary Teaching Hospital, UNESPFCAV, for human and material resources.

\section{References}

1 Dewey CW, Da Costa RC. Myelopathies: Disorders of the Spinal Cord. In: Dewey CW, Da Costa RC, eds. Practical Guide to Canine and Feline Neurology. 3rd edition Oxford: Wiley BlackWell; 2016: 329-403

2 Pool RR, Carrig CB. Multiple cartilaginous exostoses in a cat. Vet Pathol 1972;9(05):350-359

3 Friedenberg SG, Vansteenkiste D, Yost O, et al. A de novo mutation in the EXT2 gene associated with osteochondromatosis in a litter of American Staffordshire Terriers. J Vet Intern Med 2018;32(03): 986-992

4 Czerwik A, Olszewska A, Starzomska B, et al. Multiple cartilaginous exostoses in a Swiss Mountain dog causing thoracolumbar compressive myelopathy. Acta Vet Scand 2019;61(01):32-37

5 Faik A, Mahfoud Filali S, Lazrak N, El Hassani S, Hajjaj-Hassouni N. Spinal cord compression due to vertebral osteochondroma: report of two cases. Joint Bone Spine 2005;72(02):177-179

6 Bhatti S, Van Ham L, Putcuyps I, De Bosschere H, Polis I, Van Goethem B. Atlantoaxial cartilaginous exostosis causing spinal cord compression in a mature Bernese mountain dog. J Small Anim Pract 2001;42(02):79-81

7 Shores A, Tepper LC. A modified ventral approach to the atlantoaxial junction in the dog. Vet Surg 2007;36(08):765-770

8 Fingeroth JM. Dorsal Cervical Decompression (Laminectomy/ Hemilaminectomy and Laminotomy). In: Shores A, Brisson B, eds. Current Techniques in Canine and Feline Neurosurgery. New Jersey: John Wiley \& Sons Inc; 2017:484-501

9 Silver GM, Bagley RS, Gavin PR, Kippenes H. Radiographic diagnosis: cartilaginous exostoses in a dog. Vet Radiol Ultrasound 2001;42(03):231-234

10 Platt SR, Costa RC. Cervical Vertebral Column and Spinal Cord. In: Johnston SA, Tobias KM, eds. Veterinary Surgery: Small Animal. 2nd edition St Louis: Elsevier; 2018:1327-1451 